\title{
Propuesta metodológica para la renovación de los sistemas y procesos de administración de los mercados eléctricos: el caso colombiano
}

\author{
Lilliam Urrego* \\ Miguel Ángel Cañas* \\ Juan Camilo Arbeláez $z^{* * *}$ \\ Adrián Ceballos ${ }^{* * * *}$
}

Recibido: 31/03/2015 • Aceptado: 30/06/2015

\begin{abstract}
Resumen
El mercado eléctrico ha evolucionado en Colombia desde 1995, fortaleciéndose institucionalmente, dinamizando su desarrollo, y enfrentando grandes desafíos. Los sistemas de administración, que soportan el funcionamiento del mercado, han evolucionado a la par, aunque algunos se encuentran aislados entre sí o han sido desarrollados en plataformas diferentes, lo que dificulta la sostenibilidad y el mantenimiento, y reducen la facilidad de incorporar cambios.
\end{abstract}

Al referenciarse con otros mercados en el mundo, se observa gran dinámica en la incorporación de tecnologías en información y comunicaciones, avances regulatorios o requerimientos de clientes. Se requiere una renovación tecnológica que conserve el conocimiento adquirido, y permita incorporar fácilmente las tendencias del mercado.

Este artículo presenta los resultados de la propuesta metodológica para la renovación tecnológica enmarcada en el proyecto Colciencias CNBT 833559938649 de investigación tecnológica, Sistema para la administración del mercado de energía eléctrica en Colombia, Fase I.

Palabras clave: renovación tecnológica, mercados de energía, BPMN, sistemas de administración

$\mathrm{PhD}$ (c) Ing. Industria y Organizaciones; XM S. A.-E. S. P., Calle 12 sur N.ํ 18-168, Medellín (Col); tel. 5743157969; liurrego@xm.com.co

** MSc Sistemas y Computación; XM S. A. E. S. P., Calle 12 sur N. ${ }^{\circ} 18-168$, Medellín (Col); tel. 574- 3157966; macanas@xm.com.co

*** MSc Strategic Project Management; XM S. A.-E. S. P., Calle 12 sur N. ${ }^{\circ}$ 18-168, Medellín (Col); tel. 574- 3157892; jarbelaez@xm.com.co

${ }_{* * * *}$ MSc Engineering and Policy Analysis; XM S. A.-E. S. P., Calle 12 sur N. ${ }^{\circ} 18-168$, Medellín (Col); tel. 5743170989, aceballos@xm.com.co 


\title{
Methodological proposal for systems and electricity markets management processes renewal: the colombian case
}

\begin{abstract}
The electricity market has been developed in Colombia since 1995, has strengthened institutionally, its development has been dynamic, and also has solved different difficulties. Management systems, that support the market administration, have evolved following this development, however some of these are isolated from each other, or have been developed on different platforms making it difficult to ensure sustainability, maintenance and to incorporate changes.
\end{abstract}

As referenced with other markets in the world, great dynamics is observed in incorporating information and communication technologies, regulatory developments or customer requirements. A technological renovation that preserves the acquired knowledge is required, and that allows easily incorporate the development trends of the market.

This article presents the results of the proposed methodology for technological renovation framed in Colciencias CNBT 833559938649 technological research project, in management system for electric power market in Colombia, Phase I.

Key words: Technological renovation, energy markets, BPMN, Systems Management 


\section{INTRODUCCIÓN}

El mercado de energía eléctrica en Colombia comienza su operación el 20 de julio de 1995, a partir de la promulgación de las leyes 142 y 143 de 1994 que corresponden a la Ley de Servicios Públicos Domiciliarios y la Ley Eléctrica, respectivamente. En sus 20 años de evolución, el mercado se ha fortalecido institucionalmente con la CREG (Comisión de Regulación de Energía y Gas), UPME (Unidad de Planeamiento Minero Energética), CNO (Consejo Nacional de operación) y el CAC (Comité Asesor de Comercialización), y se han desarrollado en el mercado principios como la libre competencia, la desintegración vertical, la separación de actividades, las transacciones internacionales y los modelos de cargo por capacidad y confiabilidad para condiciones críticas de hidrología. Se han enfrentado fenómenos climáticos de El Niño y La Niña, además de los ataques a la infraestructura, con su impacto en la administración del mercado.

El desarrollo del mercado eléctrico en Colombia ha sido muy dinámico debido a los cambios en la regulación y a las lecciones aprendidas de la administración y la operación del mismo. Estos cambios paulatinos han llevado a que los procesos y los sistemas que soportan la administración de este mercado hayan sido implementados de acuerdo con las necesidades cambiantes. Actualmente, tanto los aplicativos como la infraestructura soportan la administración del mercado frente a la regulación vigente; sin embargo, algunos de tales aplicativos se encuentran aislados entre sí o están al final de su vida útil debido a la tecnología usada, lo que dificulta la sostenibilidad, encarece el mantenimiento y reduce su capacidad de respuesta ante nuevos cambios en el mercado. Adicionalmente los desarrollos de software y hardware también han presentado una evolución dinámica.

Las tendencias en los mercados de energía incluyen aspectos como mayores transacciones en menos tiempo, la relación de la administración del mercado con otros mercados como los financieros y el del gas, un papel más activo de la demanda en el mercado, la generación distribuida, la incorporación de nuevas tecnologías en la canasta energética como las renovables, además del intercambio inteligente de información asociado con las tecnologías de redes inteligentes.

Estas tendencias tienen implicaciones en la administración de mercados como el manejo de altos volúmenes de datos, el intercambio de información del mercado cada vez más cerca del tiempo real, el aumento de la flexibilidad de los sistemas para adaptarse a los cambios regulatorios propios de la evolución de los mercados, y la posibilidad de hacer simulaciones de implementación de cambios en los esquemas de mercado o del desempeño financiero de los agentes. Otras implicaciones son una mayor accesibilidad para los agentes para incentivar la autogestión 
apalancados en el desarrollo de nuevas tecnologías; incrementar la seguridad de los sistemas ante los riesgos de ciberataques; incrementar la monitorización y los reportes ágiles y a la medida para incentivar la competencia entre los agentes del mercado.

Para continuar prestando el soporte a la administración del mercado de electricidad, incorporar las nuevas tendencias en mercados y hacer frente a los retos de la administración, es fundamental contar con procesos y herramientas tecnológicas eficientes, un sistema que garantice la integralidad de los nuevos desarrollos tecnológicos, así como la versatilidad y flexibilidad para enfrentar los nuevos cambios regulatorios. Es así como el operador y administrador del mercado colombiano pone en marcha el proyecto SAM (Sistema de Administración del Mercado), con el fin de realizar la renovación tecnológica de los sistemas del mercado de energía, de manera integrada y que sean flexibles.

\section{METODOLOGÍA}

La propuesta metodológica para la renovación tecnológica de los sistemas de administración de los mercados de energía eléctrica consta de cuatro partes, tal como se ilustra en la figura 1. La primera, mediante la educción de los requisitos, captura el conocimiento y produce mejoras en los procesos; además, permite dar una respuesta ágil a los cambios regulatorios y a la evolución del mercado de electricidad. En la segunda sección se propone una búsqueda de tendencias de desarrollo en otros mercados en el mundo, con el fin de conocer sus experiencias e identificar aspectos importantes en la implementación de estos sistemas. En la tercera fase, se desarrolla la alineación estratégica del negocio y el desarrollo de la plataforma tecnológica de la administración del mercado teniendo en cuenta los retos y las tendencias de los mismos y, por último, las estrategias de implementación con los sistemas existentes después de ejecutar el RFP (Request For Proposal).

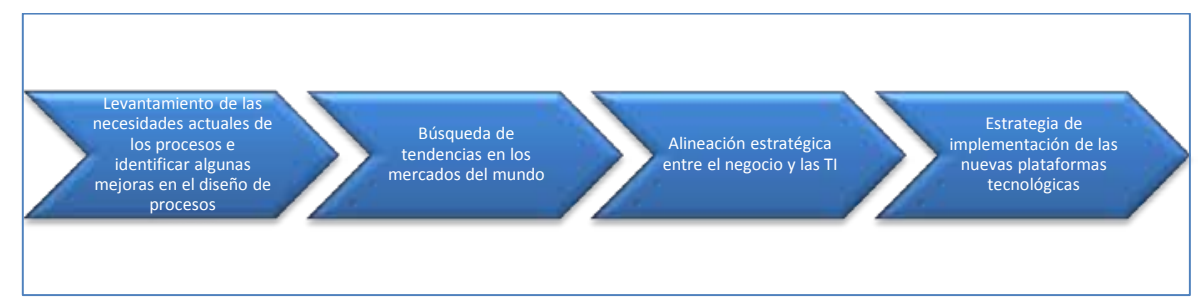

Figura 1. Metodología propuesta para la renovación tecnológica en el mercado eléctrico de Colombia Fuente: elaboración propia 


\section{EDUCCIÓN DE REQUISITOS DE LOS PROCESOS DE ADMINISTRACIÓN DEL MERCADO}

Para la gestión de los procesos se utilizan los conceptos asociados a la Gestión de Procesos de Negocio BPM (Business Process Management), que consiste en un conjunto de métodos y tecnologías estructuradas que consideran que los procesos son activos que la organización usa para crear valor a los clientes [1]. El enfoque de procesos que prima sobre las aplicaciones, áreas de negocio o tareas independientes, y permite tener una visión sistemática y holística que apoya la gestión unificada de procesos de negocio, personas y tecnología como una forma de alcanzar y mejorar los resultados empresariales.

Los procesos son transversales a toda la organización y tienen intercambio de información con distintas áreas internas y externas. De hecho, en la actualidad, los procesos en su mayoría son sistemas abiertos, complejos y que se desarrollan en parte fuera de la empresa. La información fluye de forma horizontal y automatizada, los procesos son guiados por reglas de negocio, y el control y el desempeño se hacen basados en indicadores medibles en forma oportuna.

Con este enfoque, se captura el conocimiento por medio de la comprensión del funcionamiento de los procesos, que permite mejorarlos continuamente (competitividad, rediseños, reingeniería, etc.) además de automatizarlos, monitorizarlos (con indicadores de gestión y rendimiento) e integrarlos con la infraestructura de TI. En este sentido BPM es un enfoque que permite sustituir el conocimiento escrito (procedimientos y normas) en modelos gráficos y visuales de alto nivel holístico, así como las relaciones entre procesos y el intercambio de información. En la figura 2 se muestra el diagrama de alto nivel de los procesos asociados a la administración del mercado de electricidad en Colombia.

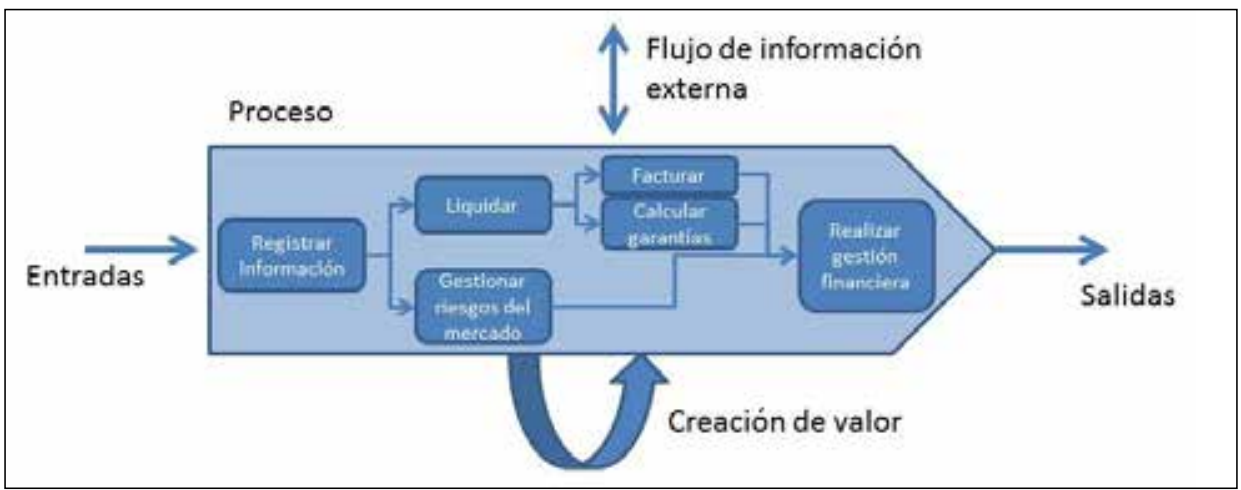

Figura 2. Esquema de alto nivel de los procesos de administración de mercado en Colombia Fuente: elaboración propia 
En el análisis de los procesos para el mercado colombiano se ejecutan dos actividades, obtención de las necesidades actuales de los procesos de negocio e identificación e implementación de mejoras en el diseño de los procesos de administración de mercados.

\section{A. Modelado de las necesidades actuales de los procesos}

La comprensión del proceso se realiza a través del modelado de los requisitos y la identificación de las necesidades del sistema, con el uso de metodologías para la valoración de adherencia y cumplimiento de procesos de negocio, la identificación de los atributos de calidad asociados, además de la determinación clara de los objetivos estratégicos del negocio y los procesos del negocio que se quieren elicitar. Posteriormente, para cada proceso se deben identificar las actividades detalladas que lo componen y definir las reglas de negocio, las variables empleadas y las relaciones con otros procesos, que permiten posteriormente identificar las necesidades del negocio y establecer los atributos de calidad y los casos de uso del proceso. Las actividades detalladas le dan al modelo la flexibilidad que se requiere para incorporar los cambios necesarios propios del mercado.

Se utilizan dos herramientas: el BPMN (Business Process Model and Notation) para modelar los procesos de negocio en una notación estandarizada que sea entendible por todos los participantes del negocio y los proveedores. Esta notación crea un puente para cerrar la brecha existente entre el diseño e implementación de los procesos [2]. En otros procesos se usa, además, UML (Unified Modeling Language), cuya notación facilita la especificación, visualización y documentación de los modelos de sistemas de software, que, sin embargo, no implica programar directamente debido a que es un lenguaje de modelado que describe en detalle casos del futuro sistema [3]. El uso integrado de los estándares BPMN y UML apalanca la obtención de resultados satisfactorios para modelar los procesos, renovar o implementar nuevos sistemas de software [4].

\section{B. Identificación de mejoras en los procesos}

Para la identificación de mejoras, se hace uso de la regulación, que la CREG ha definido en su función como regulador del sector de energía y gas, y que es aplicable a los diferentes procesos que son necesarios para la administración del mercado de energía eléctrica. A partir de esta regulación, y con el fin de garantizar su cumplimento, se procede a desarrollar las mejoras sobre las actividades de los procesos correspondientes para que generen valor y permitan eliminar la burocracia innecesaria, además de simplificar los procesos y reducir tiempos. 
Los procesos de la administración del mercado fueron mejorados en varios aspectos como la agregación de valor frente al cumplimiento regulatorio, la eliminación de reprocesos en especial en las verificaciones intermedias, la simplificación en los procesos al hacer revisiones holísticas, y en los tiempos con la estimación del cumplimiento de los objetivos al apalancarse en la tecnología y en el cumplimiento regulatorio.

También se puede hacer uso de metodologías de optimización de procesos, Lean Six Sigma (LSS) o Theory of Constraints (TOC), para disminuir el tiempo actual de los procesos y para que se garantice el cumplimiento de los requisitos de entrega de sus productos con la calidad esperada. En el caso LSS, se hacen las líneas de natación (Swim lanes) de los procesos involucrados, las cuales consisten en unos diagramas que permiten asignar responsables a las diferentes actividades dentro del proceso; se miden tiempos de estos procesos, y se identifican mejoras y optimizaciones en estos diagramas.

\section{SISTEMAS DE ADMINISTRACIÓN EN OTROS MERCADOS ELÉCTRICOS EN EL MUNDO}

Para analizar las características de los sistemas de administración en los mercados eléctricos, se llevan a cabo tres actividades. La primera es una vigilancia tecnológica con el CIDET (Centro de Investigación y Desarrollo Tecnológico), como parte del proyecto del Sistema de Administración de Mercado (SAM) en Colombia, correspondiente a una búsqueda de amplio espectro. La segunda es un referenciamiento, evaluando las principales características de los mercados considerados avanzados, con la identificación de algunos asuntos importantes para comparar. Y la tercera es una misión comercial a Estados Unidos, con el acompañamiento del USTDA (United States Trade and Development Agency), con el fin de conocer las experiencias en la implementación de sistemas de administración de mercado (Market Management Systems_MMS) y el papel que tienen los reguladores para incentivar la mejora continua en estos mercados a partir de los avances tecnológicos.

\section{A. En la vigilancia tecnológica}

La vigilancia tecnológica se hace con el fin de identificar tendencias y patrones en los sistemas de administración de los mercados eléctricos. Para esto se recopila y analiza la información de la gestión de mercados de energía, haciendo énfasis en las características del mercado colombiano. Basado en el uso de mapas conceptuales, se definen fuentes de información para la búsqueda de patentes y artículos e información no estructurada, la cual es analizada usando minería de datos [5]. La administración de los mercados de energía, a pesar de constituir un tema relevante para el sector eléctrico y de gran dinamismo en el desarrollo, no cuenta con abundante literatura de respaldo. 
Esta vigilancia tecnológica se realiza mediante la búsqueda de información estructurada en la que se encuentran bases de datos como ScienceDirect, Scopus, IEEE Xplore Digital Library, Scielo Computers and Applied Sciences Complete y EBSCO; y de información no estructurada compuesta por documentos e información disponible en la web que dan luces frente a temas de carácter comercial. De esta revisión se identifican 40 operadores de mercado en el mundo, en los cinco continentes, con un predominio de Europa y Estados Unidos en la administración de mercados regionales. En el nivel institucional se revisaron 141 publicaciones científicas entre el año 1999 y el 2013 de un total de 1500 que fueron encontradas inicialmente y que se filtraron con el fin de obtener las publicaciones más relevantes. De esta revisión se concluye que la entidad con mayor número de artículos de investigación publicados es PJM, como operador de gran parte del mercado eléctrico del Este de los Estados Unidos, quien ha venido mostrando un liderazgo en la administración de mercados; aparecen también KEMA, como firma consultora de energía eléctrica en Holanda; SAP como proveedor de software empresarial, y ABB como proveedor de sistemas para la administración de mercados eléctricos, las cuales participan normativa y tecnológicamente en la evolución de la operación de los mercados de acuerdo con las propuestas realizadas en sus publicaciones. Por países, Estados Unidos muestra el mayor número de publicaciones con 26, seguido por China con 23; se encuentran también algunas publicaciones de los países europeos y de Australia. Las publicaciones de América Latina, y en particular de Colombia, son escasas.

Respecto a las patentes, su dinámica frente a la gestión de mercados de energía es muy baja, posiblemente debido a que las tecnologías en desarrollo de métodos, procesos y software en aplicaciones para plataformas de gestión de información y algoritmos de optimización cambian rápidamente por un mejoramiento continuo, lo que dificulta cumplir con los criterios de novedad e inventiva para la obtención de la patente [5].

Se identifican posibles proveedores de sistemas para la administración de mercados en el mundo, que proveen soluciones ajustables a diversos clientes con geografías y regulaciones distintas entre los que se encuentran ALSTOM, ABB-Ventyx, Siemens, Indra, Unicorn, Gridspeak, PSI, Endimensions y Nexant entre otros. Las principales conclusiones de este trabajo se destacan a continuación:

- Los proveedores de sistemas para la gestión del mercado son diversos, proporcionan soluciones ajustables a las necesidades de los clientes, lo que les permite atender diversidad de clientes por ubicación geográfica y diferente regulación.

- Existe un trabajo conjunto para construir o mejorar los sistemas para la administración del mercado. Con base en la vigilancia tecnológica realizada, se puede inferir que las 
relaciones se encuentran regidas por ubicación geográfica y que, dada la tendencia de integración de los sistemas, se presentan trabajos colaborativos entre proveedores, operadores y generadores o transmisores de energía.

- El país líder en el tema de sistemas de gestión de mercados es Estados Unidos, seguido de China. Estos dos países tienen una gran extensión territorial y poblacional, lo que es coherente con su necesidad de mejorar sus procesos y sistemas de administración de mercados de energía eléctrica; además, los países europeos y Australia también tienen publicaciones en estos temas.

- La tendencia tecnológica es hacia mayores transacciones en menos tiempo, incrementando las frecuencias, la integración de los sistemas y la medición directa en los activos del usuario final y de los generadores.

\section{B. En el referenciamiento}

Los mercados de energía eléctrica en el mundo han venido evolucionando a un ritmo cada vez mayor por la creciente incorporación de las nuevas tecnologías en información y en comunicaciones dentro del sector eléctrico. Esta evolución ha llevado a que estos mercados cuenten con transacciones cercanas al tiempo real; respuesta de la demanda ante señales de precios; mayor integración con otros mercados, mercados regionales; administración del riesgo a través de cámaras de compensación, y una mayor convergencia con otros sectores económicos como los de telecomunicaciones, transporte, financiero, entre otros.

Los cambios y retos que se presentan en la oferta y la demanda en estos mercados se han enfocado no solo hacia su diseño sino también hacia el mejoramiento tecnológico. Desde la oferta, se esperan mercados con una alta participación de la generación distribuida y de fuentes alternativas de generación que permitan la reducción de emisiones y que, a su vez, sustituyan el uso de combustibles fósiles, mientras que por el lado de la demanda, se espera, además, un incremento de soluciones significativas hacia una mayor eficiencia e inteligencia en el consumo de energía, que le proporcionen más opciones de elección al usuario final con señales de precios justos en tiempo real para la toma de decisiones.

Los mercados eléctricos han hecho avances importantes en la regulación, los sistemas y los procesos. En sistemas y procesos se han implementado los Sistemas de Administración de Mercado (Market Management Systems $-M M S-$ ), los cuales se han caracterizado por una gran adaptabilidad que les ha permitido mantener un flujo eficiente de la información en los procesos y entre los diferentes agentes del mercado, mejorar los tiempos de ejecución de los procesos y facilitar los cambios en software y hardware. 
Los MMS son sistemas diseñados para administrar las transacciones entre proveedores de energía y empresas distribuidoras mediante la operación de procesos estandarizados, y su respectiva validación y seguimiento [6]. Si bien los procesos asociados con la administración dependen de las reglas de cada país, guardan entre sí similitudes propias de los mercados.

Algunos autores como Silvia-Monroy [7] relacionan las funciones de los MMS de liquidación del mercado diario, despacho económico, y servicios auxiliares, interactuando con sistemas como administración de energía (Energy Management SystemEMS), Sistemas de Información Comercial (Commercial Information system - COMS) y Sistemas de Información de Mercado (Market Information Systems - MIS). Hay un conjunto de funciones que son desempeñados por un MMS que se implementan de acuerdo con las reglas del mercado, y dependen de los modelos de los recursos y sistemas basados en información del sistema y del mismo mercado.

Algunos proveedores de estos sistemas tienen sus propias definiciones de los MMS. Ventyx [8], por ejemplo, define un conjunto de aplicaciones avanzadas y de infraestructura moderna de Tecnologías de Información (TI) que apoya en un gran rango todas las funciones relacionadas con el mercado que son desempeñadas por administradores del mercado mundial.

En el mundo existen casos exitosos de implementación de estos sistemas, en los que se incluye el rediseño de los procesos de mercado, así como nuevas plataformas tecnológicas que permiten la interacción con participantes del mercado y con otros sistemas de información e incorporan los nuevos desarrollos de los mercados eléctricos. Como ejemplo de estos mercados y de las metodologías usadas para la implementación de estos sistemas cabe mencionar los casos de PJM [9, 10, 11], ERCOT [12], CAISO (Estados Unidos) [13], OMIE (España y Portugal) [15], East China Power Market (China) [16], entre otros.

PJM, el operador de gran parte del mercado del este de los Estados Unidos, al desarrollar el nuevo diseño de su mercado, empleó un proceso colaborativo para establecer los sistemas que le permitieran una operación eficiente, teniendo como reto la creación de un mercado que se acomodara a las necesidades de varios de sus jugadores y de sus usuarios. Esto llevó a que fuese necesario implementar los procesos y sistemas que se ajustaran a estos requerimientos. Su sistema es conocido como MSRS (Market Settlement Reporting System), el cual permite a sus usuarios tener acceso y descargas de informes en línea, y a los miembros del mercado, un ingreso a sus cuentas, la verificación del estado de sus garantías y facturas, y les permite la descarga de reportes personalizados de acuerdo con sus necesidades. 
ERCOT (Electric Reliability Council of Texas), el cual administra el mercado de energía eléctrica en Texas, cuenta con herramientas tecnológicas de información que se clasifican en tres categorías: operaciones técnicas, operaciones comerciales y bodegas de datos. Las operaciones técnicas incluyen el Sistema de Administración del Mercado (MMS) que trabaja en conjunto con el Sistema de Operación del Sistema (POS). Las operaciones comerciales incluyen el registro de clientes, cargas de perfiles, adquisición y carga de datos, liquidación, facturación y transacciones. El MMS consiste en una interfaz web que facilita la interacción con los participantes del mercado y la base de datos en la que el sistema del mercado reside. Dado que el mercado de ERCOT está en constante evolución, ya que aparecen nuevas reglas de mercado y los estándares de confiabilidad son establecidos periódicamente, es necesario que las herramientas de TI sean actualizadas continuamente para reflejar estos cambios. De esta manera, para mantener la eficiencia del mercado y la confiabilidad del sistema, se requiere que el sistema de ERCOT trabaje en conjunto con todos los demás sistemas en una forma confiable y segura todo el tiempo, por lo que un nuevo cambio en los procesos está bien coordinado al usar un modelo estructurado de migración, el cual debe ser aprobado mediante un protocolo que analiza el impacto que este cambio tiene en diferentes módulos del sistema.

El nuevo sistema para el mercado de electricidad en CAISO (California ISO) tiene un componente de mercado diario y otro de tiempo real. Adicionalmente, cuenta con un nuevo sistema de subastas y de liquidación que fueron adquiridos de diferentes proveedores, lo que representó un reto grande en integración. Para lograr asumir este reto, CAISO usó tecnología SOA (Service Oriented Architecture) y adoptó el estándar IEC CIM como una plataforma de comunicación. De esta forma, al usar el estándar IEC CIM se crearon mensajes en SOA, no solo para comunicarse entre los diferentes sistemas nuevos sino también para integrarlos con los sistemas que tenían en ese momento.

\section{En la misión comercial}

En la misión comercial se destacan aspectos del mercado que deberían considerarse en la gestión de este tipo de desarrollos tecnológicos, entre los cuales se encuentra:

- Los mercados de energía en el mundo cuentan con diferentes diseños, lo cual implica que los procesos y sistemas de administración de mercados deban adaptarse a las diferentes reglas de negocio que son propias de cada mercado.

- Se deben ajustar y definir muy bien los requisitos para el diseño de los nuevos procesos y su implementación en los sistemas que los soportan. Lo anterior incluye una descripción detallada de las funcionalidades requeridas. 
- Es indispensable tener procesos y sistemas que puedan adaptarse a los cambios regulatorios, ya que las reglas de los mercados cambian frecuentemente y, por tanto, se requiere tener alta flexibilidad para no incurrir en grandes costos en el momento de hacer ajustes.

- Los requerimientos de los procesos son críticos; por lo tanto, es importante contar con una prospectiva que indique hacia dónde se estará moviendo tanto el mercado como la regulación. Esto para tenerlo en cuenta dentro del diseño de los nuevos procesos y de los actuales.

- La arquitectura de información debe ajustar los nuevos modelos a la evolución de los mercados; esto implica un esfuerzo grande en la migración de los datos a los nuevos desarrollos.

- Los desarrollos tecnológicos implementados en otros mercados del mundo tienen la capacidad de administrar grandes volúmenes de información de manera eficiente

\section{RENOVACIÓN DE LA PLATAFORMA TECNOLÓGICA}

Esta propuesta metodológica está basada en el enfoque de los procesos de negocio, que los integra de forma transversal a la estructura tecnológica con el fin de generar valor a los clientes. Desde el punto de vista del proceso se requiere que la gestión del conocimiento y la dinámica del desarrollo se vean reflejadas en el modelo de requisitos, el cual tiene como finalidad tener una mayor comprensión de las necesidades del sistema y delimitar su alcance para tener una renovación tecnológica que apalanque el funcionamiento actual del proceso y el desarrollo futuro del mismo. Desde el punto de vista tecnológico se requiere que la solución se integre con los sistemas de información y en la infraestructura definida en forma coherente. Este concepto se ilustra en la figura 3.

Al disponer de un modelo BPMN actual de los procesos en especial en el flujo de información junto con el diseño mejorado de los procesos, además de la revisión de los mercados en el mundo que identifican aspectos importantes a tener en cuenta en los diferentes desarrollos de los sistemas de información que se pueden implementar, es posible realizar una definición de la estrategia de implementación de las nuevas plataformas tecnológicas, de nuevos procesos y una propuesta de un modelo de alineación del negocio con las tecnologías de información.

Para lograr la alineación se puede desarrollar la solución o adquirirla en el mercado. En este último caso los proveedores deben tener experiencia y se desarrollan con ellos las actividades locales propias de la regulación de cada país. La gestión de procesos en algunos de los mercados se hace en las herramientas con la notación BPMN, y 
podrían usar una BPMN suite, para desarrollar una aplicación cercana (con ajustes y revisiones) a la funcionalidad modelada en el proceso de negocio.

En términos generales, las características de los mercados son similares en la estrategia de negocio, aunque difieren en la implementación en los mercados locales. Algunos le dan mayor prioridad a la interacción entre los sistemas de operación o el manejo de clientes, muy supeditado a la reglamentación de cada mercado.

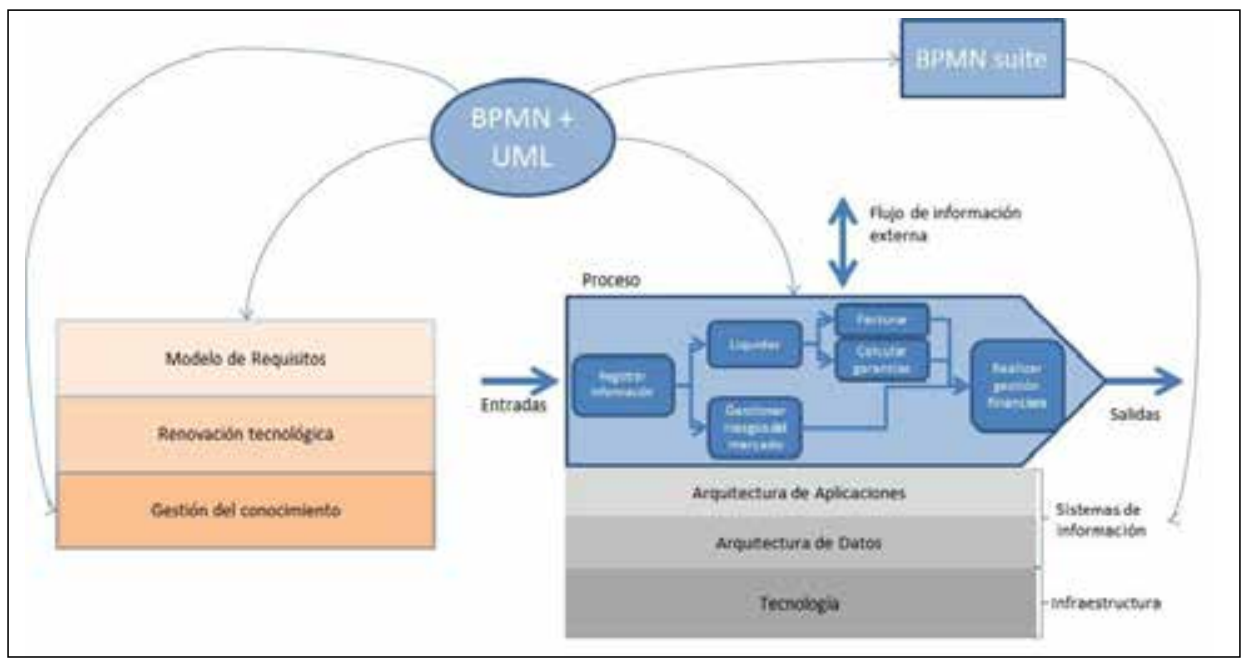

Figura 3. Estrategia de selección de la plataforma tecnológica Fuente: elaboración propia

En última instancia, para determinar si estos sistemas y el flujo de información en los procesos pueden ser tenidos en cuenta más allá de una referencia, es necesario mantener una articulación entre el negocio y las tecnologías de información [14]. De esta manera se espera que esta alineación incluya aspectos como: indicadores de cubrimiento de los requisitos de los procesos en la plataforma tecnológica, indicadores de costo para mantener la alineación del negocio con TI, uso de modelos adicionales a BPMN para mejorar la especificación de sistemas en el mercado, indicadores de eficiencia para la educción de requisitos, al igual que indicadores de calidad de los modelos para ser automatizados.

\section{ESTRATEGIA DE IMPLEMENTACIÓN DE LA PLATAFORMA TECNOLÓGICA}

La estrategia propuesta considera cuatro frentes de trabajo: solución del sistema, integración con sistemas externos, migración de datos y pruebas. Al implementar esta estrategia, se debe evaluar, monitorizar, supervisar y realizar la gestión, además de asegurar la integridad de la arquitectura que resulte de la solución implementada por 
sus proveedores, así como las integraciones realizadas con los sistemas legados y las interfaces utilizadas. Esta estrategia de implementación se presenta en la figura 4.

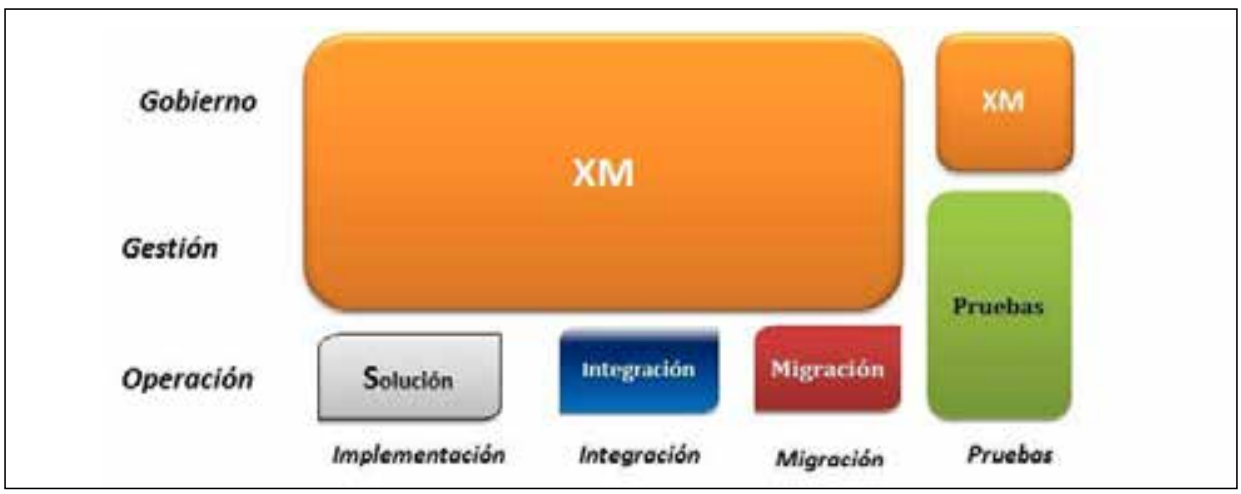

Figura 4. Estrategia para la implementación de nuevas plataformas tecnológicas en el mercado de energía en Colombia

Fuente: elaboración propia

a. La solución del sistema corresponde a la implementación, parametrización y licenciamiento de un sistema de administración de mercado (MMS) que cubra los diferentes requisitos de los nuevos procesos diseñados, además de la infraestructura requerida para que este sistema funcione adecuadamente.

b. La integración con sistemas externos al MMS busca garantizar que el nuevo sistema se integre efectivamente con aplicativos existentes tanto para la administración del mercado como para los usados en la operación del sistema. El funcionamiento del mercado no debe afectarse en forma negativa.

c. En la migración se busca que los datos sean transferidos de una manera gradual y efectiva desde los sistemas anteriores al nuevo sistema. Se debe tener especial cuidado con las características asociadas a la seguridad de la información.

d. Las pruebas se basan en la realización de las pruebas funcionales, que aseguren un desempeño efectivo de la solución en los diferentes procesos para la administración del mercado de energía.

\section{CONCLUSIONES Y RECOMENDACIONES}

La propuesta metodológica encuentra que la alineación estratégica, el conocimiento y la dinámica del desarrollo del proceso de negocio se ven reflejados en el modelo de requisitos para la renovación tecnológica, apalancando el funcionamiento actual y su desarrollo futuro. Tecnológicamente, la solución se integra con los sistemas de información y en la infraestructura actual en forma coherente y confiable. 
La captura del conocimiento, al tener un enfoque de proceso de negocio, con una representación visual y holística de las actividades, las relaciones internas y externas así como el flujo de información, hace que se incremente la facilidad al cambio y la coherencia en las ocasiones que sea necesario modificarlos, al incluir las variaciones en la regulación o del desarrollo del mercado.

En los mercados analizados, los Estados Unidos tienen un liderazgo en la implementación de sistemas de administración de mercados, incorporando las decisiones de los clientes y participantes, así como las nuevas tendencias en el desarrollo del mercado. Se encuentran varios proveedores de tecnología exitosos en el desarrollo de estos sistemas.

La solución a implementar trae beneficios en cuanto al manejo de grandes volúmenes de información, actualización de los aplicativos ante nuevas tecnologías de hardware o de desarrollo de software y demás de la coordinación de los sistemas y plataformas homologadas, que son flexibles y aseguran la calidad de la información del mercado. En la estrategia de implementación, se hace énfasis en la integración con aplicaciones existentes, a la migración de los datos y las pruebas de la solución para asegurar la continuidad del proceso de negocio.

Se recomienda incrementar el mejoramiento de procesos usando metodologías y herramientas especializadas, para avanzar más en la optimización de los mismos.

\section{REFERENCIAS}

[1] J. País, "BMPN (Business Process Management) Como alcanzar la agilidad y eficiencia operacional a través de BPM y la empresa orientada a procesos", [En linea]: BPMteca, 2013.

[2] B. V. 2.0, «www.omg.org,» Object Management Group_OMG, 2011. [En línea]. Available: http://www.omg.org/spec/BPMN/2.0/PDF. [Último acceso: 11 2014].

[3] «OMG,» 2005. [En línea]. Available: http://www.omg.org/gettingstarted/what_is_uml.htm. [Último acceso: 2015].

[4] M. e. a. López, «Modelling using UML and BPMN the integration of open reliability, maintenance and condition monitoring management systems:an application in an electric transformer system.,» Computers in industryJournal, vol. 64, n. ${ }^{\circ}$ 5, pp. 524-542, 2013.

[5] L. Niebles y G. Gabriela, «CIDET_Identificación de Tendencias de tecnologías para el desarrollo de un sistema de información para la administración de mercado de electricidad colombiano Vigilancia tecnológica Colombia,» CIDET, Medellin, 2014.

[6] M. Ratnakaran, C. Grossardt y C. Robertson, «Market Management System.,» Patent Application Publication, 2007/0112579. USA. 2007. 
[7] C. Silva-Monroy y J.-P. Watson, «"Integrating Energy Storage Devices Into Market Management Systems",» Proceedings of the IEEE, vol. 102, n. ${ }^{\circ}$ 7, pp. 1084-1093, 2014.

[8] Ventyx, «Ventyx,» 2014. [En línea]. Available: Ventyx. Enable e_cient market and system opewww.ventyx.com/mwg-internal/de5fs23hu73ds/progress?id=T3qadkxD2MlYrABFdAH k8CiNviOQW7vvWFVxinKklnc. [Último acceso: 2014].

[9] PJM, «Market Settlement Reporting System (MSRS).,» 2014. [En línea]. Available: http:// www.pjm.com/markets-and-operations/etools/msrs.aspx. [Último acceso: 2014].

[10] Z. Fan, T. Horger y J. Bastian, «“Current and emerging challenges in PJM energy market,”,» de Transmission and Distribution Conference and Exposition, 2010 IEEE PES, New Orleans, LA, USA, 2010.

[11] Z. Fan, T. Horger, J. Bastian y A. Ott, «"An overview of PJM energy market design and development,",» de ,Electric Utility Deregulation and Restructuring and Power Technologies, 2008. DRPT 2008. Third International Conference on, Nanjuing China, 2008.

[12] S. Sundhararajan, A. Boecker, J. Dondeti, R. Howard, J. Tamby, S. Grendel y A. Jayantilal, « "Experience with ERCOT System's IT development and operation,”,» Power Systems, IEEE Transactions on, vol. 18, n. ${ }^{\circ}$ 2, pp. 535,540, 2003.

[13] E. Haq, D. Haller, K. Rahman y B. Iverson, «"Use of Common Information Model (CIM) in Electricity Market at California ISO,”,» de Power and Energy Society General Meeting, 2011 IEEE, San Diego CA, 2011.

[14] J. Luftman, «Assessing Business-It Alignment Maturity,» Communications of AIS, vol. 4, n. ${ }^{\circ}$ 14, pp. 1-46, 2000.

[15] Y. ZHANG y e. al, «Experience with East China Power Market IT Development and Operation,» de Power System Technology, 2006. PowerCon 2006. International Conference on IEEE, 2006., Chongqing, China, 2006.

[16] OMIE, «OMIE,» 2014. [En línea]. Available: http://www.omel.es/files/mu_interfazam_3.pdf . [Último acceso: 2014]. 